\title{
EXACT SOLUTION OF LOSSY ASYMMETRICAL COUPLED DIELECTRIC SLAB WAVEGUIDES
}

P. R. Young* and R. J. Collier ${ }^{\dagger}$

*Electronic Engineering Laboratories, University of Kent at Canterbury, Canterbury, Kent, CT2 7NT, UK. email: p.r.young@ukc.ac.uk ${ }^{\dagger}$ Microelectronics Research Centre, Cavendish Laboratory, University of Cambridge, Madingley Road, Cambridge CB3 0HE, UK. email: rjc48@ cam.ac.uk

\section{ABSTRACT}

This paper gives an exact characteristic equation for asymmetrical coupled dielectric slab waveguides with losses in both the guiding and surrounding regions. For the lossless case the solution of a single transcendental equation is all that is required for the evaluation of the propagation constant.

\section{INTRODUCTION}

There has been renewed interest in dielectric and non-radiative dielectric waveguides recently $[1,2,3]$. These structures provide very low-loss transmission and are likely to be the preferred transmission line medium for future 3D MMICs at upper mm-wave frequencies. Many dielectric waveguide components such as directional couplers [4] and filters [5] rely on composite structures for there operation. For each of these components, accurate values for the modal propagation constants are required. In general, solutions of coupled structures are obtained using coupled mode theories [6, 7]. The coupled mode is approximated by a weighted sum of the modes that exist on the isolated structures. However, these methods are only valid for large separations or well-confined modes [8]. Furthermore, coupled mode theory does not work well for asymmetrical guides [8]. Marcuse [9] presented a technique for the solution of compound slab waveguides with width and permittivity asymmetry. Unfortunately, his method is cumbersome, requiring numerical techniques to find the eigenvalue of an $8 \times 8$ determinant. In this paper, we derive an exact transcendental characteristic equation for the general asymmetrical coupled slab waveguide, the roots of which can easily be found. Furthermore, the technique can be used for the calculation of losses in coupled dielectric waveguides, which is an important consideration at mmwave frequencies.

\section{THEORY}

Consider two parallel slab waveguides, $A$ and $B$, separated by a distance $2 D$ and with thicknesses $2 a$ and $2 b$, respectively (Fig. 1). Guide $A$ occupies region 2 and has a relative permittivity $\varepsilon_{a}$. Guide $B$ occupies region 4 and has a relative permittivity $\varepsilon_{b}$. Regions 1,3 and 5 all have a relative permittivity $\varepsilon_{2}$. In general, the permittivity in each of the regions will be complex, $\varepsilon_{n}=\varepsilon_{n}^{\prime}\left(1-j \tan \delta_{n}\right)$. All five regions have permeability $\mu_{0}$. We make the usual slab assumption that all the field components are independent of $y$ and that the $z$ dependence is $\exp \left(-\gamma_{z} z\right)$, where $\gamma_{z}=\alpha_{z}+j \beta_{z}$. $\alpha_{z}$ is the longitudinal attenuation constant and $\beta_{z}$ is the longitudinal phase constant. The wave equation then reduces to a one dimensional Helmholtz equation:

$$
\frac{d^{2}}{d x^{2}} \Phi_{y}(x)+k_{x}^{2} \Phi_{y}(x)=0
$$

where $\Phi_{y}=E_{y}$ for TE modes and $\Phi_{y}=H_{y}$ for TM modes. We choose the following fields over the five regions

$$
\begin{array}{ll}
\text { 1: } & \Phi_{y}(x)=A_{1} e^{\alpha_{x 2}[x+(D+2 a)]} \\
2: & \Phi_{y}(x)=A_{2} \cos \left\{k_{x a}[x+(D+2 a)]-\phi_{a}\right\} \\
\text { 3: } & \Phi_{y}(x)=A_{3} \cosh \left[\alpha_{x 2} x\right]+A_{4} \sinh \left[\alpha_{x 2} x\right] \\
\text { 4: } & \Phi_{y}(x)=A_{5} \cos \left\{k_{x b}[x-(D+2 b)]+\phi_{b}\right\} \\
\text { 5: } & \Phi_{y}(x)=A_{6} e^{-\alpha_{x 2}[x-(2 b+D)]}
\end{array}
$$

where $A_{1} \ldots A_{6}$ are amplitude constants and $\phi_{a}$ and $\phi_{b}$ are constant phase terms. The transverse propagation constants are given by $\alpha_{x 2}^{2}=\varepsilon_{2} k_{0}^{2}-\gamma_{z}^{2}, k_{x a}^{2}=\gamma_{z}^{2}-\varepsilon_{a} k_{0}^{2}$ and $k_{x b}^{2}=\gamma_{z}^{2}-\varepsilon_{b} k_{0}^{2}$ where $k_{0}$ is the free-space wave number. For TE modes, $E_{y}$ and $H_{z}$ must be continuous at the boundaries between the different regions. For TM modes $H_{y}$ and $E_{z}$ must be continuous. Therefore, using Maxwell's equations to calculate $H_{z}\left(E_{z}\right)$, and equating $E_{y}\left(H_{y}\right)$ and $H_{z}\left(E_{z}\right)$ at the four boundaries gives eight equations for the boundary conditions of the TE (TM) modes. These equations can be combined to yield 


$$
2 D=\frac{1}{2 \alpha_{x 2}} \ln \left[\frac{\sin \left(2 k_{x a} a\right) \sin \left(2 k_{x b} b\right)}{\sin \left(2 \phi_{a}-2 k_{x a} a\right) \sin \left(2 \phi_{b}-2 k_{x b} b\right)}\right] .
$$

where $\phi_{a}=\tan ^{-1}\left(\rho_{a} \alpha_{x 2} / k_{x a}\right)$ and $\phi_{b}=\tan ^{-1}\left(\rho_{b} \alpha_{x 2} / k_{x b}\right)$ with $\rho_{a}=\rho_{b}=1$ for TE modes and $\rho_{a}=\varepsilon_{a} / \varepsilon_{2}$ and $\rho_{b}=\varepsilon_{b} / \varepsilon_{2}$ for TM modes. We see that $\alpha_{x 2}, k_{x a}$ and $k_{x b}$ are all functions of $\gamma_{z}$. Thus, the right hand side of eqn. (2) is a function of a single variable - the longitudinal propagation constant $\gamma_{z}$. Therefore if $a, b, D, \varepsilon_{a}$, and $\varepsilon_{b}$ are specified, we can solve eqn. (2) for all possible solutions of $\gamma_{z}$.

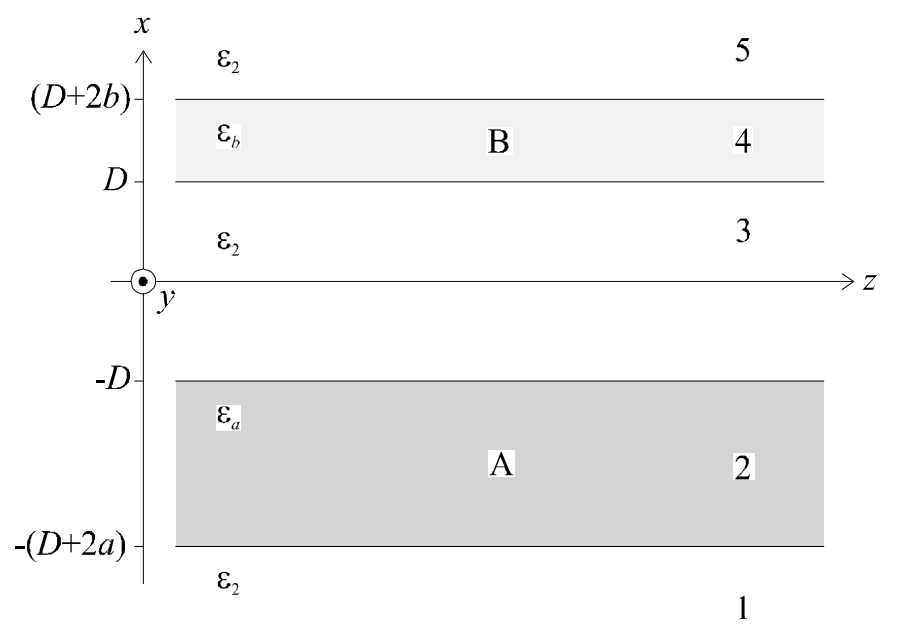

Fig. 1. Geometry of two parallel dielectric slab waveguides.

\section{RESULTS}

Fig. 2 shows the normalised separation $2 D / \lambda_{0}$ plotted against the normalised propagation constant $\beta_{z} / k_{0}$ for different $2 a /$ $\lambda_{0}$ ratios. Both guides are identical, i.e. $a=b$ and $\varepsilon_{a}=\varepsilon_{b}=2.07$. From Fig. 2 we see that, for the given $2 a / \lambda_{0}$ ratios, there are two solutions to eqn. (2). The solution with the largest value of $\beta_{z}$ corresponds to the lowest order (even) mode. The solution with the smallest value of $\beta_{z}$ corresponds to the next higher order (odd) mode. This can be readily seen be substituting $\beta_{z}$ into the field equations. It should be noted that for further increases in frequency, higher order modes would propagate. However, in general, coupled structures are limited to the two-mode case. We clarify Fig. 2 with an example. For a structure with $a=b=0.5 \mathrm{~mm}, 2 D=6.0 \mathrm{~mm}$ and $\lambda_{0}=10.0 \mathrm{~mm}$, Fig. 2 shows that two modes exist: an odd mode with $\beta_{z} / k_{0}=1.012$ and an even mode with $\beta_{z} / k_{0}=1.068$. Solutions with values of $\beta_{z} / k_{0}$ approaching unity correspond to modes near to low frequency cut-off. It is seen that in the low frequency case, $2 a / \lambda_{0}=0.1$, the odd mode is cut-off until the guide separation $2 D$ is larger than $0.4 \lambda_{0}$. As expected the even and odd mode propagation constants tend to the value of the propagation constant of the isolated guides for increasing separation.

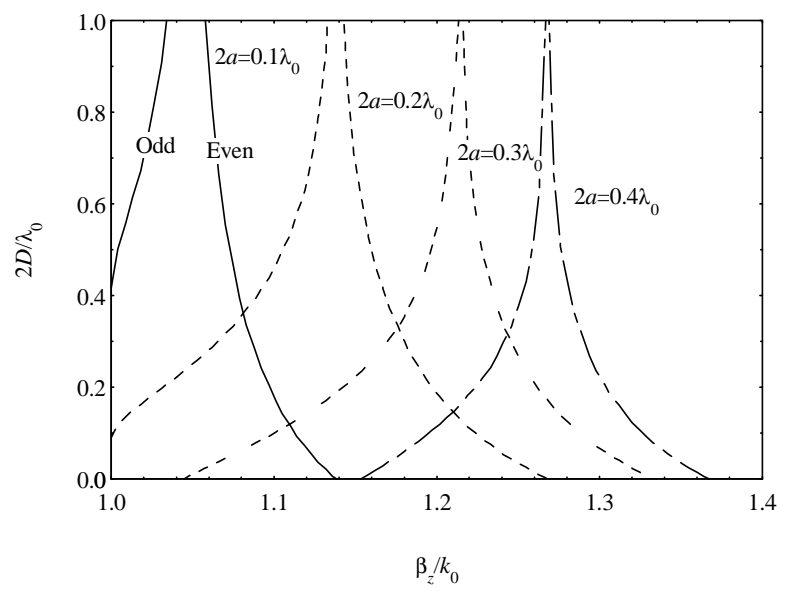

Fig. 2. Normalised separation $2 D / \lambda_{0}$ against $\beta_{z} / k_{0}$ for several different $2 a / \lambda_{0}$ ratios. $2 a=2 b$ and $\varepsilon_{a}=\varepsilon_{b}=2.07$.

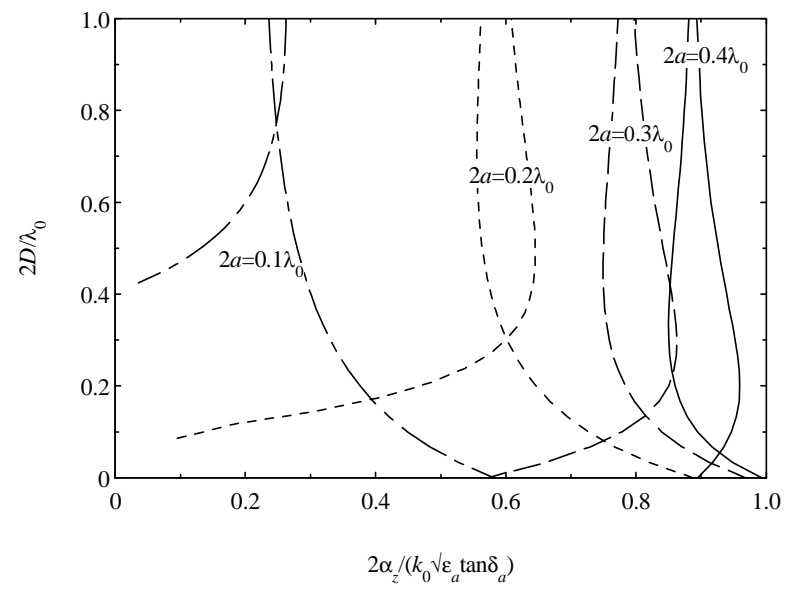

Fig. 3. Normalised attenuation constant for TE mode symmetrical coupled slab waveguide. $2 a=2 b, \varepsilon_{2}=1$, $\varepsilon^{\prime}{ }_{a}=\varepsilon^{\prime}{ }_{b}=2.07$ and $\tan \delta_{a}=\tan \delta_{b}=3.0 \times 10^{-4}$ 
For completeness Fig. 3 shows the attenuation constant $\alpha_{z}$ for varying separation with $2 a=2 b, \varepsilon_{2}=1, \varepsilon^{\prime}{ }_{a}=\varepsilon^{\prime}{ }_{b}=2.07$ and $\tan \delta_{a}=\tan \delta_{b}=3.0 \times 10^{-4}$. Notice that the odd mode solutions increase with $2 D$, reach a maximum and then decrease slightly as they converge to the isolated value. For the even modes, $\alpha_{z}$ decreases as $2 D$ increases, reaches a minium and then increases with $2 D$, finally converging to the isolated value. This means that for some values of $2 D$ the even mode of the coupled structure has a lower attenuation constant than that of the isolated dielectric waveguide. This may have some implications for low-loss propagation.

Once the propagation constant is determined from eqn. (2) the values of $\alpha_{x 2}, k_{x a}, k_{x b}, \phi_{a}$ and $\phi_{b}$ can be calculated. Thus, by applying the appropriate boundary conditions the field amplitude constants $A_{1} \ldots A_{6}$ can be evaluated. Fig. 4 shows field plots for the even and odd modes of a TE guide for $2 a=\lambda_{0} / 2$ and $\varepsilon_{a}=\varepsilon_{b}=2.0$. Fig. 4 (a), (b) and (c) show the symmetrical case with $2 D=\lambda_{0} / 4,2 D=\lambda_{0} / 2$ and $2 D=\lambda_{0}$, respectively. Fig. 4 (d), (e) and (f) are for the same guide separations as above but with a width asymmetry of $a / b=2$. Similarly, Fig. 4 (g), (h) and (I) are for $a / b=4$ and Fig. 4 (j), (k) and (l) are for $a / b=8$.
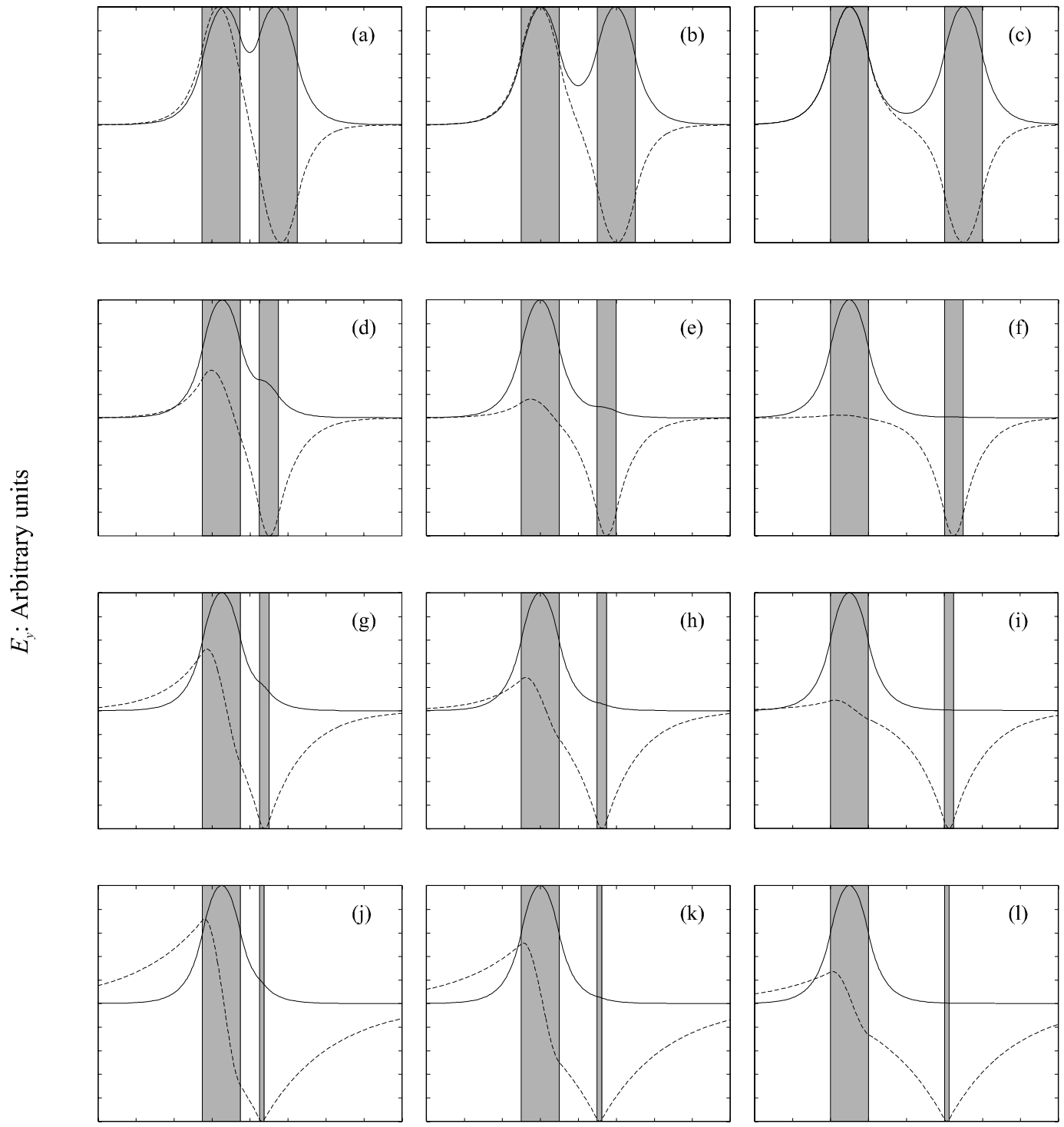

$$
x / \lambda_{0}
$$

Fig. 4. Effects of separation and width asymmetry on the modal electric field profiles for TE coupled slab waveguide. Solid lines show the even modes, dashed lines show the odd. 


\section{CONCLUSIONS}

In summary, an exact transcendental characteristic equation has been presented for both the TE and TM solutions of asymmetrical coupled slab waveguides with width and permittivity asymmetry. The solution of this new equation is simpler than previous methods and, unlike coupled mode theories, is exact. The technique is therefore valid for all guide separations and asymmetries. Furthermore, the technique can be used to calculate the attenuation constant when dielectric losses are present, which is often the case at millimetre-wave frequencies.

\section{REFERENCES}

[1] Dalaire, J. and Wu, K.: "Complete characterization of transmission losses in generalized nonradiative dielectric (NRD) waveguide," IEEE Trans Microwave Theory Tech., vol. 48, no. 1, January 2000, pp. 121-125.

[2] Boone, F. and Wu, K.: "Mode conversion and design consideration of integrated nonradiative dielectric (NRD) components and discontinuities," IEEE Trans Microwave Theory Tech., vol. 48, no. 4, April 2000, pp. 482-492.

[3] Tang, J. and Wu, K.: "Co-layered integration and interconnect of planar circuits and nonradiative dielectric (NRD) waveguide," IEEE Trans Microwave Theory Tech., vol. 48, no. 4, April 2000, pp.519-524.

[4] Ikäläinen, P. K. and Matthaei, G.: "Design of broad-band dielectric waveguide 3-dB couplers," IEEE Trans Microwave Theory Tech., vol. MTT-35, no. 7, July 1987, pp. 621-628.

[5] Matthaei, G. L., Park, D. C., Kim, Y. M. and Johnson, D. L.: "A study of the filter properties of single and parallelcoupled dielectric-waveguide gratings," IEEE Trans Microwave Theory Tech., vol. MTT-31, no. 10, October 1983, pp. 825-835.

[6] Marcuse, D.: "The Coupling of Degenerate Modes in Two Parallel Dielectric Waveguides," Bell Syst. Tech. J., Vol. 50, No. 6, pp 1791-1816, July-August 1971.

[7] Snyder, A. W.: "Coupled-mode Theory for Optical Fibers," J. Opt. Soc. Am., Vol. 62, No. 11, pp. 1267-1277, November 1972.

[8] Hardy, A. and W Streifer, W.: “Coupled Mode Theory of Parallel Waveguides," J Lightwave Tech., Vol. LT-3, No. 5, pp 1135-1146, October 1985.

[9] Marcuse, D.: "Directional couplers made of nonidentical asymmetric slabs. Part I: Synchronous couplers," $J$. Lightwave Tech., vol. LT-5, no. 1, January 1987, pp. 113-118. 